\title{
Welfare of farmed fish in present and future production systems
}

\author{
Anders Kiessling • Hans van de Vis • \\ Gert Flik · Simon Mackenzie
}

Received: 2 December 2011/Accepted: 16 December 2011/Published online: 14 January 2012

(C) Springer Science+Business Media B.V. 2012

The effort manifested in this special issue, dedicated to the welfare of farmed fish, is the joint result of members of the Cost action 867 "Welfare of Fish in European Aquaculture." This publication is supported by COST. COST is a framework for European Cooperation in the field of Scientific and Technical Research, which facilitates the coordination of nationally funded research on a European level. For the

Hans van de Vis-Wageningen Aquaculture is a consortium of IMARES (Institute for Marine Resources \& Ecosystem Studies) and AFI (Aquaculture and Fisheries Group,

Wageningen University), both part of Wageningen University \& Research Centre (WUR).

\section{A. Kiessling $(\square)$}

Departments of Wildlife, Fish, and Environmental Studies, Swedish University of Agricultural Sciences, Umeå, Sweden

e-mail: Anders.kiessling@slu.se

H. van de Vis

IMARES, Wageningen UR, P.O. Box 77, 4400 AB Yerseke, The Netherlands

G. Flik

Department of Animal Physiology, Institute for Water and Wetland Research, Faculty of Science, Radboud University Nijmegen, Heyendaalseweg 135, 6525 AJ Nijmegen, The Netherlands

\section{S. Mackenzie}

Institut de Biotecnologia i de Biomedicina, Universitat Autonoma de Barcelona, 08193 Bellaterra,

Barcelona, Spain coordination and harmonization of national and EU level research project efforts, a network was established. The network comprised 26 countries, including Canada, New Zealand and Australia, and more than 100 researchers. The action began in 2006 and continued for 5 years, ended with a final meeting in Madrid, Spain, in March 2011. The main aim of the action was to provide a meeting platform supporting (a) discussion and information between active researchers, (b) harmonization throughout Europe of the Welfare concept and (c) formulation of tomorrow's production systems introducing welfare as a management tool.

During the time of the action, a tremendous development in perception, understanding and implementation of welfare as a concept has occurred. At the start of the action, the main focus was on disease as well as water and feed quality, that is, variables with very direct effects on growth. Now, at the end of the action, further variables have been introduced including fish preferences and biological-based stimulation measures. Furthermore, a palpable shift from disease toward prophylactic health has been evident, that is, preventive measures included when farmers discuss welfare. The action as such is in no way responsible for this alone, but has been one contributing factor, contributing to the perception that welfare may set the biological limits for a sustainable production system. In other words, compromising welfare would indicate a production format violating the biological capacity of the animal and thereby demanding nonsustainable 
counter actions such as increased medication, shorter life spans and acceptance of disease.

One could very grossly divide actions to support the welfare of farmed fish into two main approaches. The first concerning actual production systems and how to secure welfare, and the second concerns emerging production systems. Today's intensive aquaculture systems are surprisingly similar often independent of the cultured species and location and could be divided in a several major types. This can be explained by a tradition of technology-driven development in combination with the absence of economic incentives beyond quantity and guaranteed delivery. The question arising at the end of the action is "If biological driven development is the most coherent approach will intensive culture systems, based on the biological needs/abilities of specific species/breeds, be economically viable?" Thus if specific welfare measures (e.g. fish preference) are integrated into production practice could they be economically viable potentially via a higher retail price. Many examples from terrestrial farmed animals indicate that systems promoting the natural biological production potential of the animal, if correctly handled, may outperform systems based upon solely technical solutions. The present special issue, being a co-production between the Cost 867 network and Fish Physiology and Biochemistry summarizes not only the present status of the field but also formulates a scientific platform for future production systems, putting the animal in the center of the debate.

This special issue is divided into four sections:

- Conceptual frame work of welfare in fish

- Factual component of welfare

- Assessment of welfare in fish (including some experimental papers)

- Implementation of welfare in fish

The papers are either opinionated reviews or research articles with a general interest and broad approach.

In the first section, the question is asked whether fish are the victim of "speciesism," that is, is there a different ethical framework for fish compared to other animals in the eyes of society. This section also includes articles discussing the fundamentals of welfare and whether controlled laboratory experiments have a direct relevance to the multivariable reality at the farm level.

The second section includes reviews about risk analysis as a tool to quantify welfare and thereby enable comparison of different factors affecting welfare. Also several members of the action have made a very serious attempt to distinguish between veterinarian medicine as an indicator of bad welfare and as a prerequisite for good welfare, that is, how does one distinguish between the two sides of the coin. Another group has approached genomics and welfare, and yet another the issue of nutrition and welfare, resulting in probably the most upto-date and comprehensive written source of welfare on these subjects available today. Welfare at slaughter and Welfare implications of anesthesia are two other reviews dealing with two very common procedures during both production and research. Cortisol is probably one of the most common welfare indicators especially in applied research. The review addressing cortisol does not only deal with cortisol as indicator for physiological response but also its relationship to psychological condition. Also behavioral indicators and injuries/lesions as objective tools to assess welfare in fish have been addressed as two joint activities of the action and are presented here. Fish live in a protein and fat-dominated energy food chain, in contrast to carbohydrate-driven nutrition in mammals. Nitrogen balance and quality in feed may be an important factor for nutritional welfare in fish and is also reviewed within this special issue.

The more specific research articles include a range of relevant subjects including recovery from surgical procedures, a major interest for any researcher interested in a viable animal when using in situ experiments. Dietary composition and its implications for welfare is yet another question of major interest questioning whether dietary choice and its economics are essential and the implications of a "single diet" in aquaculture, that is, the animals only choice is to eat more or less, as the pre-prepared diet is formulated from an anthropocentric vision of what we think is best for fish. Other subjects included in this section include the importance of the timing of feeding in relation to the natural behavioral rhythm of fish and the effects of live chilling. The issue is then rounded off by a socio-behavioral reflection upon a series of practical activities to implement welfare at farm level together with the farmer.

\section{The organisation COST}

COST- the acronym for European Cooperation in Science and Technology- is the oldest and widest 
European intergovernmental network for cooperation in research. Established by the Ministerial Conference in November 1971, COST is presently used by the scientific communities of 36 European countries to cooperate in common research projects supported by national funds.

The funds provided by COST - less than $1 \%$ of the total value of the projects - support the COST cooperation networks (COST Actions) through which, with EUR 30 million per year, more than 30000 European scientists are involved in research having a total value which exceeds EUR 2 billion per year. This is the financial worth of the European added value which COST achieves.

A "bottom up approach" (the initiative of launching a COST Action comes from the European scientists themselves), "à la carte participation" (only countries interested in the Action participate), "equality of access" (participation is open also to the scientific communities of countries not belonging to the European Union) and "flexible structure" (easy implementation and light management of the research initiatives) are the main characteristics of COST. As precursor of advanced multidisciplinary research COST has a very important role for the realisation of the European Research Area (ERA) anticipating and complementing the activities of the Framework
Programmes, constituting a "bridge" towards the scientific communities of emerging countries, increasing the mobility of researchers across Europe and fostering the establishment of "Networks of Excellence" in many key scientific domains such as: Biomedicine and Molecular Biosciences; Food and Agriculture; Forests, their Products and Services; Materials, Physical and Nanosciences; Chemistry and Molecular Sciences and Technologies; Earth System Science and Environmental Management; Information and Communication Technologies; Transport and Urban Development; Individuals, Societies, Cultures and Health. It covers basic and more applied research and also addresses issues of pre-normative nature or of societal importance.

Web: http://www.cost.eu

\section{Legal notice by the COST Office}

Neither the COST Office nor any person acting on its behalf is responsible for the use which might be made of the information contained in this publication. The COST Office is not responsible for the external websites referred to in this publication. 